\title{
Rhithrogena gorrizi Navás, 1913 et $R h$. castellana Navás, 1927 : redescription des imagos (Ephemeroptera, Heptageniidae)
}

\author{
A.G.B. Thomas' \\ M. Sartori ${ }^{2}$
}

Mots clés : Ephemeroptera, Heptageniidae, néotype, topotypes, redescriptions.

Première redescription, à partir de spécimens de la collection J. Aubert (Musée zoologique de Lausanne), des imagos $\sigma$ de Rhithrogena gorrizi et de $R$ h. castellana Navás. L'imago $\odot$ et les subimagos $\sigma$ et $q$ de $R h$. castellana sont décrites pour la premjère fois. Cette dernière espèce était tombée dans l'oubli depuis sa description originale.

La synonymie Rh. comitissa Navás $1933=R h$. gorrizi est en outre proposée.

Rhithrogena gorrizi Navás, 1913 et Rh. caslellana Navás, 1927 : new description of the imagos (Ephemeroptera, Heptageniidae).

Keywords : Ephemeroptera, Heptageniidae, néotype, topotypes, new descriptions.

New descriptions of the male imagos of Rhithrogena gorrizi and $R h$. castellana Navas, from the Aubert collection in the Zoological Museum in Lausanne, are given. The female imago and both sexes of the subimago of $R$ h. castellana are described for the first time. The latter species has been overlooked since it was originally described.

That $R h$. comitissa Navás 1933 is a junior synonym of $R h$. gorrizi is proposed.

Des espèces sud-européennes du genre Rhithrogena décrites par le révérend père L. Navás, trois seulement sont bien connues et redécrites avec une précision autorisant leur identification. Ce sont $: R h$. diaphana, $R h$. ferruginea et $R h$. loyolaea (voir respectivement Puthz 1973, Sowa 1970 et Thomas 1970).

Des séries types des autres espèces, il ne subsiste, dans le cas le plus favorable (Thomas 1968), qu'une imago $\sigma$, en plus ou moins bon état, ou bien même, à notre connaissance, plus de type du tout.

Or, à notre avis, la description détaillée de genitalia o d'Ephéméroptères ne peut être effectuée avec sécurité sur un seul individu, en raison d'une variabilité morphologique plutôt importante. Grâce à la collection du $\mathrm{D}^{\mathrm{r}} \mathrm{J}$. Aubert, déposée au Musée de Lausanne, nous sommes en mesure de redécrire

\footnotetext{
1. Laboratoire d'Hydrobiologie, UA 695 du C.N.R.S., U.P.S., 118 route de Narbonne, 31062 Toulouse Cedex, France.

2. Musée Zoologique, Place Riponne 6, 1005 Lausanne, Suisse.
}

les imagos ơ de deux autres espèces de la péninsule ibérique : $R h$. gorrizi et $R h$. castellana.

\section{1. - Rhithrogena gorrizi Navás, 1913}

Rhitrogena Gorrizi Navás, 1913, (sic), Boln Soc. aragon. Cienc, nat., $12: 61-62$, pl. V fig. 1 a et b.

Rhithrogena gorrizi Thomas, 1968, Annls Limnol., 4 (2) : 209-218, pl. I fig. 6 et 7, pl. Il fig. 6, pl. III fig. 4.

\section{Matériel examiné.}

a) Collection Navás:

Un spécimen $\sigma$, piqué à sec, qui n'appartient pas à la série type - puisque capturé en 1914 - mais provient de la localité typique (Zaragoza) à une date parfaitement compatible avec la période de vol indiquée par Navás. Cet exemplaire correspond bien à la description originale et a déjà èté désigné comme lectotype (Thomas 1968). Il est dans un état général relativement correct et son identification n'est nullement douteuse. Il porte, de la main du R.P. Navás, les indications suivantes: "Rhitrogena Gorrizi Nav. " (sic) Navás S.J. dét. et "Zaragoza 28-V-14 ". 

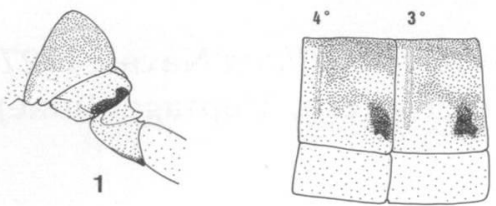

2
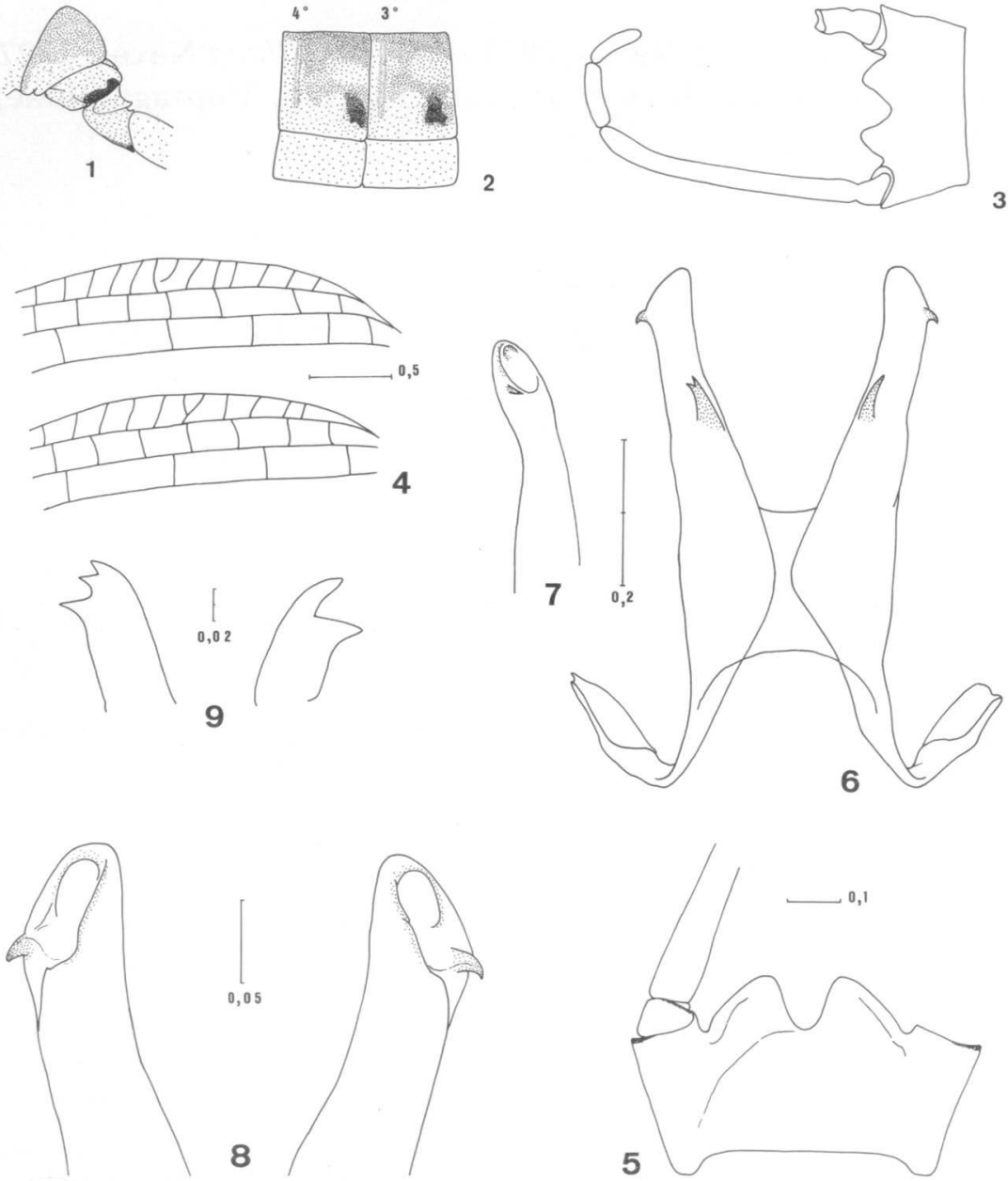

FIG. 1 à $9:$ Rhithrogena gorrizi Navás, imago ơ. Echelle en mm.

1 à 3 : lectotype ; 4 à $9:$ topotypes. $1:$ coxa et trochanter de la patte postérieure. $2: 3^{e}$ et $4^{e}$ segments abdominaux en vue latérale. 3 : styligère et styles. 4 : aire ptérostigmatique. $5:$ styligère. 6 à $8:$ pénis en vues ventrale (6), latérale (7) et dorsale (8). 9 : titillateurs. 
Les pièces suivantes font défaut : une patte antérieure, une patte médiane, les ailes postérieures et les cerques. Le scutum est un peu endommagé. Du còté droit, manquent le titillateur et la plus grande partie du style (Thomas t.c.).

Ce spécimen est déposé au Musée de Zoologie de Barcelone où est maintenant transférée la collection Navás.

b) Collection J. Aubert :

8 imagos or en alcool à $70^{\circ}$ provenant aussi de Saragosse (Zaragoza), le 23-V-1947 (J. Aubert et F. Schmid leg.). La date de capture est très voisine de celles d'une partie des exemplaires de la série type et nous désignons ces spécimens comme topotypes. Ils sont déposés au Musée Zoologique de Lausanne.

\section{Description (imago or).}

Diagnose sommaire : espèce de petite taille ; coloration d'ensemble brun moyen avec des taches foncées sur les tergites abdominaux; ailes claires, hyalines.

\section{Tête.}

Face fauve ; ocelles brun moyen cerclés à la base d'un anneau foncé, violacé noirâtre ; yeux rosâtres avec une large bande basale externe noirâtre.

\section{Thorax.}

Tergite prothoracique brun clair un peu fauve : coxas 1 plus claires, jaunâtres ainsi que la région centrale proéminente du sternite dont la périphérie est brune; pattes 1 brun assez foncé sauf les fémurs, brun moyen, porteurs d'une tache médiane noirâtre, plus longue que large, très visible et d'une seconde maculation apicale plus petite et moins intense.

Mesothorax brun moyen, le scutum un peu plus foncé que les pleures. Quelques traces de pigment sur la membrane pleurale en direction de la coxa 1 .

Metathorax et base de l'abdomen brun nettement plus foncé.

Pattes 2 et 3 plus claires que Pl, brun moyen du fémur jusqu'au tarse inclus. Les fémurs portent des taches brun noirâtre intense. Ces taches sont longues comme la largeur du férnur et étroites : elles s'étendent seulement sur le tiers ou le quart médian de cette largeur. Les coxas 2 et 3 portent près de leur bord postéro-externe une tache noirâtre bien visible au contour proximal concave (fig. 1).
Ailes presque entièrement hyalines (à peine jaunâtres dans la région basale antérieure), à nervation claire, brun jaunâtre. Aire ptérostigmatique guère plus foncée que le reste de la membrane alaire, portant une douzaine de nervures transverses pratiquement pas ramifiées (fig. 4).

\section{Abdomen.}

Brun assez foncé et plus ou moins ferrugineux dans la région moyenne des tergites. Ces derniers s'éclaircissent sur les côtés où ils deviennent fauves. Une tache brun noirâtre intense près du coin inféro-antérieur des tergites, très apparente, en particulier du $3^{e}$ inclus au $8^{e}$ inclus (fig. 2).

Cerques brun clair.

Genitalia.

Styligère (fig. 3 et 5) brun clair à bord postérieur très saillant, arrondi et profondément entaillé au milieu. Ce contour convexe a la forme d'un secteur de cercle et les bords de l'incision sont subrectilignes. Pénis (fig. 6 à 8 ) à lobes très longs, étroits, tronqués obliquement vers l'extérieur à l'apex. Chaque lobe porte une assez forte épine marginale externe et un titillateur long et étroit, terminé par deux pointes, plus rarement une seule ou trois (fig. 9).

Taille.

Longueur de l'aile antérieure du lectotype: $7,4 \mathrm{~mm}$, des spécimens de la collection Aubert : 6,5 à $7,2 \mathrm{~mm}$.

\section{2. - L'identité de Rhithrogena comitissa Navás, 1933}

Rhithrogena comitissa Navás, 1933, Rvta Acad. Cienc. exact. fis.- quim. nat. Zaragoza, $16: 17-19$, fig. 3.

Parmi les espèces du genre Rhithrogena décrites par le R.P. Navás, et jamais retrouvées depuis, se trouve $R$ h. comitissa, dont il n'existe d'ailleurs plus de type. La localité typique de cette espèce (Sobradiel) est très proche de celle de $R h$. gorrizi : elle est aussi située sur l'Ebre, à $16 \mathrm{~km}$ seulement en amont de Saragosse. En outre, les dates de capture des spécimens des deux espèces sont proches dans l'année.

$R h$. comitissa et $R h$. gorrizi appartiennent aussi au même groupe d'espèces par la morphologie des genitalia $\sigma$ et par la taille. Les différences essentielles entre les deux descriptions du père Navás résident dans la coloration. En particulier, la figure de 


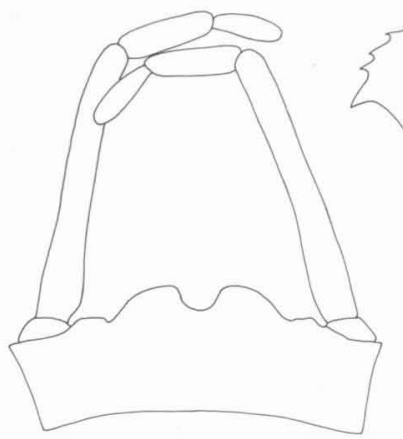

10

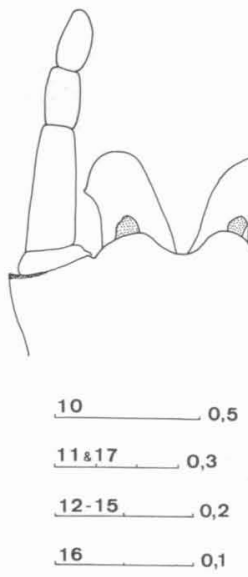

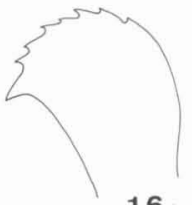

$16 a$
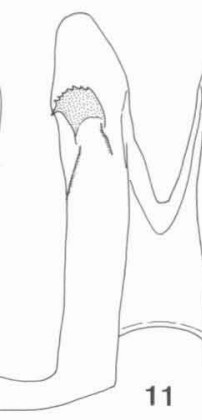

11
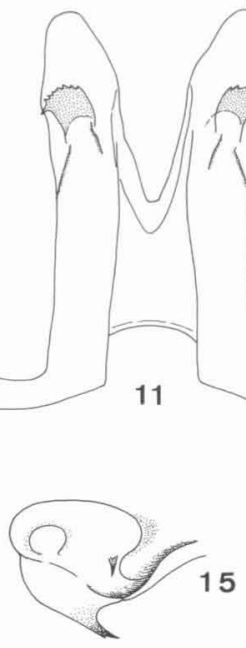

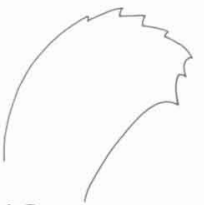

16 b
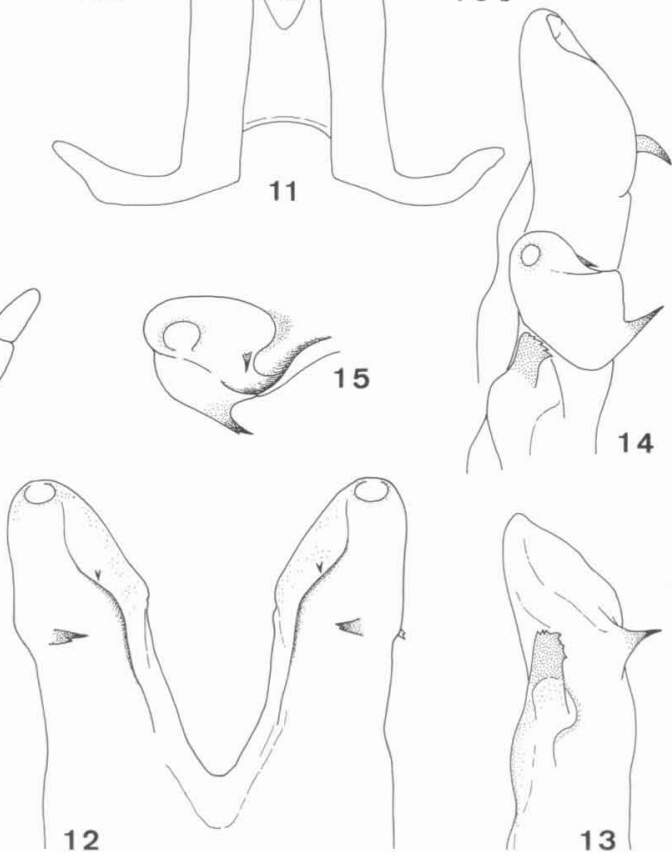

13

FIG. 10 à 17 : Rhithrogena castellana Navás, imago $\sigma$ néotype (10 à 16) et subimago ơ (17). Echelle en mm. 10 : styligère et styles (entre lame et lamelle). 11 à 15 : pénis en vues ventrale (11), dorsalé (12), latérale (13), latéro-apicale (14) et apicale (15). 16 : titillateurs. 17 : genitalia du subimago en vue ventrale. 
genitalia $\sigma$ relativement bien effectuée pour $R h$. comitissa est applicable à $R h$. gorrizi. Nous interprétons done $R h$. comitissa comme une variation seulement plus foncée de $R h$. gorrizi.

Les présents individus de $R h$ gorrizi de la collec. tion Aubert avaient d'ailleurs déjà été examinés par le $\mathrm{D}^{\mathrm{r}} \mathrm{V}$. Puthz (Schlitz) : ils ont été déterminés par lui comme appartenant à l'espèce $R h$. comitissa, ce qui explique probablement la mention de ce nom spécifique dans la seconde édition de la Limnofauna Europaea (Puthz, 1978).

En conséquence, nous proposons la synonymie suivante :

Rhithrogena comitissa Navás, $1933=R h$. gorrizi Navás, 1913.

\section{3. - Rhithrogena castellana Navás, 1927.}

Rhithrogena castellana Navás, 1927, Revta Acad' Cienc. exact. fis.quim. nat. Zaragoza, 10 : 106-108, fig. 5.

\section{Matériel examiné.}

Tous les exemplaires proviennent de la collection J. Aubert ; ils sont conservés en alcool à $70^{\circ}$ et sont déposés au Musée Zoologique de Lausanne :

- 1 imago $\sigma$ provenant de Cercedilla (localité typique): rio de la Venta à $1200 \mathrm{~m}$, le 27-V-1954.

-1 i. $\sigma, 9$ i,,, 2 s.i. $\sigma$ et 1 s. i. 9 provenant de Ventorillo: rio Navalmedio vers $1400 \mathrm{~m}$, le 27-V-1954.

Navás a décrit cette espèce grâce à une récolte de J. M. Dusmet effectuée " a orillas del rio Guadarrama " (Dusmet 1927). En réalité, ce cours d'eau est formé, nettement en aval de Cercedilla, par la confluence du rio de la Venta et du rio de las Puentes dont le principal tributaire est le rio Navalmedio.

Comme celui de $R h$. comitissa, le type de $R h$. castellana a été recherché en vain par l'un de nous (Thomas 1968). Il est probablement perdu ( $D^{r}$ F. Espanol et R.P. Palazon Delattre, comm. verb. et in litt.). Nous désignons comme néotype l'imago o* provenant de Ventorillo $(4,5 \mathrm{~km}$ de Cercedilla) et non le topotype, pour son meilleur état général de conservation.

\section{Description.}

Imago $o$.

Diagnose sommaire : espèce de taille moyenne, de coloration générale brun jaunâtre clair, unie sur l'abdomen. Ailes à nervures transverses très pigmentées formant un reticulurn net dans le tiers basal antérieur.

Tête.

Face jaune grisâtre, scape brun gris ; ocelles gris bleuâtre cerclés de brun foncé à la base. Yeux de teinte chair avec une large bande basale externe bleu noirâtre.

Thorax.

Prothorax brun jaunâtre sale. Scutum jaune assez clair ainsi qu'une grande partie des pleures: scutellum et metathorax brun moyen, de même que le sternite du mesothorax.

Pałtes antérieures brun moyen, rapport des longueurs du tarse 1 au tibia $1: 1,45$ à 1,66 . Pattes 2 et 3 jaune clair assez uniforme à l'exception de la pointe apicale du trochanter, et de l'extrême apex du fémur, brun. Apex des tarses et des griffes brun grisâtre.

Ailes antérieures hyalines sur la plus grande partie de leur surface sauf le tiers basal jaune brun plus ou moins foncé; nervures longitudinales claires, brun jaunâtre ; nervures transverses brunes et bordées d'un liséré brun violacé intense dans la moitié proximale de l'aile jusqu'à R2, conférant à cette région un aspect nettement réticulé. Aire ptérostigmatique blanc grisâtre, peu marquée, avec une vingtaine de nervures transverses plus ou moins ramifiées selon les individus.

Ailes postérieures à nervation presque transparente dans la région postéro-inférieure ; tiers basal antérieur jaune brun.

Abdomen.

Coloration dorsale jaune à fauve, plutôt translucide sur les flancs. Sternites jaunes sauf le $9^{c}$, légèrement brun près des pleures.

Cerques brun grisâtre à jointures claires, s'éclaircissant vers l'apex.

Genitalia.

Styligère brun jaunâtre, styles brun moyen à base élargie. Protubérances du styligère (fig. 10) assez marquées, plus ou moins nettement asymétriques (saillie maximale vers l'intérieur) et séparées par une profonde incision arrondie, demi-circulaire. L'ensemble n'est pas plan mais concave. Pénis (fig. 11 à 15) à deux lobes développés, larges et longs, dont la courbure apicale est pratiquement symétrique. 
Une forte denticulation dorso-externe, orientee transversalement vers l'intérieur et une autre, interne, plus petite, dorsale, dans la gouttière pénienne. Titillateurs larges et courts, multidentés (fig. 16).

\section{Imago $\%$.}

Caractères distinctifs par rapport au $\sigma$ :

Coloration d'ensemble encore plus claire; aile antérieure pratiquement hyaline sur toute sa longueur; reticulum formé par les nervures transver. ses brun violacé, moins marqué mais encore bien visible : meso et metathorax plus clairs : cerques gris jaunâtre.

Subimagos of et $\%$.

Face et funicule antennaire brun grisâtre. Yeux uniformément bleu noirâtre.

Traces d'insertions musculaires sur le pronotum très apparentes. Jointures des sclérites bien marquées sur le mesonotum.

Coloration des ailes gris moyen uniforme.

Pattes jaune grisâtre avec une tache médiofémorale gris violacé à noirâtre de plus en plus intense et étendue, de $P_{1}$ à $P_{3}$.

Genitalia $\alpha$ : fig. 17.

Taille.

Longueur de l'aile antérieure : $12,5 \mathrm{~mm}$ pour les ơ, 12,5 à $14 \mathrm{~mm}$ pour les $\%$.

Longueur des cerques : environ $25 \mathrm{~mm}$ pour les $0,17 \mathrm{~mm}$ pour les $\$$.

Ces valeurs sont pratiquement identiques à celles relevées par Navás.

A l'exception du catalogue bibliographique des Ephéméroptères de la péninsule ibérique (Alba Tercedor 1981), la seule citation de $R$ h. castellana depuis sa description originale est donnée par Sowa (1984). Ce dernier classe l'espèce - uniquement sur la base de cette description sommaire - dans le groupe semicolorata. Or, $R h$. castellana présente des affinités avec le groupe sowai-buresi - en particulier avec $R h$. daterrai Sowa 1984, décrite du Portugal, dont elle est très proche ou même peut-être synonyme.
Remerciements

C'est pour nous un plaisir que de remercier le Professeur P. Goeldlin et le Dr D. Cherix pour avoit mis à notre disposition la collection J. Aubert, déposée au Musée Zoologique de Lausanne, ainsi que le R.P. L. Palazon Delattre (Colegio del Salvador) qui nous a aimablement prété, pour une longue duréte, le dernier spécimen de $R h$. gorrizi de la collection Navás.

Nous sommes reconnaissants au Dr J. Alba (Grenade) pour l'aide bibliographique apporté lors de la recherche de la localité typique de $R h$. castellamo.

\section{Travaux cités}

Alba Tercedor (J.). 1981 - Recopilacion de citas de Efemeropteros en la peninsula iberica e islas Baleares. Trab. Monogr. Dep. Zool. Univ. Granada, (N.S.), 4 (2) : $41-81$.

Dusmet (J.M.). 1927. - In Seccion bibliographica. Boln R. Soc. esp. Hist. nat., $27: 303.304$.

Navás (L.). 1913. - Notas entomologicas. 4. Excursiones por los alrededores de Zaragoza. Boln Soc. aragon. Cienc. nat., 12 : $61-69+$ pl. V.

Navás (L.). 1927. - Mis excursiones cientificas en 1926. Revta Acad. Cienc. exact. fis.-quim. nat. Zaragoza, 10:81-124.

Navás (1.). 1933. - Faunula de Sobradiel (Zaragoza). Ibid., 16: 87.120.

Puth? (V.). 1973. - Was ist Baetis aurantiaca Burmeister 1839 (Ephemeroptera, Insecta)? Philippia, 1 (5) : 262-270.

Puthz (V.). 1978. - Ephemeroptera in : J. Illies, Limnofauna Europaed. pp. 256-263. Fischer Verlag. Stuttgart.

Sowa (R.). 1970 (1971), - Sur la taxonomie de Rhithrogena semicolorata (Curtis) et de quelques especes voisines d'Europe continentale (Ephemeroptera : Heptageniidae). Rev. suisse Zool. 77 (4) : 895-920.

Sowa (R.). 1984. - Contribution à la connaissance des espèces européennes de Rhithrogena Eaton (Ephemeroptera, Heptagenijdae) avec le rapport particulier aux espèces des Alpes et des Carpates. Proc. IV th Int. Conf. Epherneroptera, V. Landa et al. eds, $\operatorname{CSAV}: 37-52$.

Thomas (A.). 1968. - Quelques Ecdyonurus et Rhithrogena euro péens de la collection Navás (Ephemeroptera). Anmls Limnol., 4 (2) : 209-218.

Thomas (A.). 1970. - Sur la taxonomie de deux Rhithrogena des Pyrénees (Epherneroptera, Heptageniidae). Annls Limnol., 6 (3) : 305.315. 